\title{
Iron-Manganese Bimetallic-Organic Framework as a Photocatalyst for Degradation of Rhodamine B Organic Dye Under Visible Light
}

\author{
Tran Thi Kim Ngan ${ }^{1,2}$, Tran Bich Thuy ${ }^{3}$, Lam Van Tan ${ }^{1,2}$, Trung Thanh Nguyen ${ }^{4,5, *}$ \\ ${ }^{1}$ Institute of Environmental Sciences, Nguyen Tat Thanh University, Ho Chi Minh City, Viet Nam. \\ ${ }^{2}$ Faculty of Environmental and Food Engineering, Nguyen Tat Thanh University, Ho Chi Minh City, Viet Nam. \\ ${ }^{3}$ Institute of Environmental Science, Engineering and Management, Industrial University of Ho Chi Minh City, \\ Viet Nam. \\ ${ }^{4}$ Nanomaterial Laboratory, An Giang University, 18 Ung Van Khiem St., Dong Xuyen Dist, Long Xuyen City, An \\ Giang Province, Viet Nam. \\ ${ }^{5}$ Vietnam National University Ho Chi Minh City, Linh Trung Ward, Thu Duc District, Ho Chi Minh City, Viet \\ Nam.
}

Received: $18^{\text {th }}$ July 2021; Revised: 29th September 2021; Accepted: 30 th $^{\text {th }}$ September 2021 Available online: 2nd October 2021; Published regularly: December 2021

\section{Abstract}

In recent years, there have been many research works on use of different methods to treat textile dyeing wastewater such as mechanical, biological and chemical methods (using oxidizing agents, such as: $\mathrm{H}_{2} \mathrm{O}_{2}, \mathrm{O}_{3}$, and $\mathrm{H}_{2} \mathrm{O}_{2} / \mathrm{O}_{3}$ ). However, some traditional textile dyeing wastewater treatment methods such as mechanical and biological methods have limitations in treating these pollutants thoroughly. To enhance the treatment efficiency, the use of photocatalysts combination with strong oxidizing agents, such as $\mathrm{H}_{2} \mathrm{O}_{2}$, has been extensively developed in recent years. In this study, the iron-centred bimetallic organic framework Fe-MOF has been synthesized by partial replacement of $\mathrm{Fe}^{3+}$ ions with $\mathrm{Mn}$ metal ions by solvent-thermal method. The analytical methods used to evaluate the structural characterization of the as-synthesized materials including Scanning Electron Microscope (SEM), Brunaurer-Emmett-Teller (BET), X-ray Diffraction (XRD), Fourier Transform Infra Red (FT-IR), and UV-Vis Diffuse Reflectance Spectroscopy (DRS). The experiments on the decomposition of organic pigment Rhodamine B were performed under varying conditions of $\mathrm{pH}$, catalyst mass and $\mathrm{RhB}$ colorant concentration. Experiments with different electron capturers indicate that $h^{+}$plays a major role in the photochemical degradation of RhB. The stability and durability of the $0.1 \mathrm{Mn} / \mathrm{Fe}-\mathrm{MOF}$ catalyst were evaluated through the leaching and recycle experiments, showing that the RhB degradation efficiency of the photocatalyst decreased modestly after five repetitions.

Copyright (C 2021 by Authors, Published by BCREC Group. This is an open access article under the CC BY-SA License (https://creativecommons.org/licenses/by-sa/4.0).

Keywords: Bimetallic-organic framework; Photocatalysis degradation; Rhodamine B

How to Cite: T.T.K. Ngan, T.B. Thuy, L.V. Tan, T.T. Nguyen (2021). Iron-Manganese Bimetallic-Organic Framework as a Photocatalyst for Degradation of Rhodamine B Organic Dye Under Visible Light. Bulletin of Chemical Reaction Engineering \& Catalysis, 16(4), 916-924 (doi:10.9767/bcrec.16.4.11764.916-924)

Permalink/DOI: https://doi.org/10.9767/bcrec.16.4.11764.916-924

\section{Introduction}

Metal-organic framework materials (MOFs) are a multidimensional spatial network, made

* Corresponding Author.

Email: ntthanh@agu.edu.vn (T.T. Nguyen);

Telp: +84-090 7101590, Fax: +84 2963842560 up of metals or metal oxides and connected by ligands of multi-organic acids into a lattice regular nanoscale with a surface area that can reach over $6000 \mathrm{~m}^{2} / \mathrm{g}$ [1-4]. Some recently published studies showed that, due to the natural pore structure of MOFs, they are used as a catalysts in a number of chemical reactions related 
to materials and pharmaceutical manufacturing technologies $[5,6]$. In addition, depending on the metal framework structure and organic ligand, the applicability of MOFs is also different [7]. With the large capillary size, which makes the diffusion and movement of substances into the capillary relatively easy, these materials have great potential for applications in the field of catalysis and adsorption [8-10]. Especially during synthesis, the physical and chemical properties of MOFs can be tuned by combining functional groups on organic bonds or on unsaturated metal sites in the lattice framework of MOFs. On the other hand, the properties of MOFs mainly depend on the metal components such as density, stability, and magnetism, etc. However, MOFs are mainly based on single metal, so the properties of MOFs will be differently adjusted if a second metal is present in the lattice framework of the MOFs [11,12].

The outstanding performance in many applications, including gas adsorption, catalysis, energy storage and conversion, and luminescence sensing of bimetallic MOFs is of recent interest to researchers [13-16]. Currently, researchers have used modified MOFs $(\mathrm{Fe})$ as photocatalyst materials with high photocatalytic efficiency and potential due to excitation under the visible light region [17-19]. Besides, to ensure sustainable development and limit the emission of organic pigments causing environmental pollution in these industries, many treatment measures have been introduced such as chemical methods physical, biological, and chemical methods. In which, the chemical method using photocatalyst active photocatalysts to decompose organic pigments is highly effective, because the products of the decomposition process are intermediate chemical substances that are less toxic [20,21]. This method usually uses a catalyst under light conditions, generating $\mathrm{HO} \cdot$ radicals has a very strong oxidizing ability, can decompose most toxic organic substances. Recently, Tang et al. [22] carried out the synthesis of iron and copper bimetallic MOFs $\left(\mathrm{Fe}_{\mathrm{x}} \mathrm{Cu}_{1-\mathrm{x}}(\mathrm{BDC})\right)$ as a novel Fenton-like catalyst prepared by a solvothermal method and its application in the catalysis of sulfamethoxazole (SMX) in the presence of hydroperoxides. Furthermore, the $\mathrm{Fe}_{0.75} \mathrm{Cu}_{0.25}$ (BDC) catalyst showed almost $100 \%$ of SMX removal within $150 \mathrm{~min}$ and good reusability for SMX degradation [22]. In addition, the publication of Gu et al. [8] also mentioned that the bimetallic FeMg-MIL-88B material was synthesized at various molar ratios by the heat transfer method. It is noteworthy that changing the molar ratio led to a change in the surface area, morphological characteristics related to the length or diameter of the crystal. With respect to the synergy from the two active metal centers, the decomposition efficiency of FeMg-MIL-88B is enhanced, reflected in the ability to remove arsenic in 5 minutes to reach $99.6 \%$ [23]. Partial replacement of ferric metal in organic frameworks with manganese has been reported by Sun et al. [24]. The results showed that the catalytic efficiency is clearly improved when $\mathrm{Mn}$ is incorporated in the decomposition of liquid phenol [26]. At the same time, in order to prevent the rapid recombination of electron $\left(e^{-}\right)$ and hole $\left(h^{+}\right)$pairs, inorganic oxidants (e.g., $\mathrm{H}_{2} \mathrm{O}_{2}, \mathrm{KBrO}_{3}$, and $\left.\left(\mathrm{NH}_{4}\right)_{2} \mathrm{~S}_{2} \mathrm{O}_{8}\right)$, act as an electron acceptor introduced in photocatalytic processes, greatly enhances the photocatalytic effect of materials [27].

Based on the outstanding properties and high catalytic efficiency of the bimetallic materials, we synthesized $\mathrm{Mn} / \mathrm{Fe}-\mathrm{MOF}$ materials at the molar ratio of $0.1 \mathrm{n}(\mathrm{Mn}): \mathrm{n}(\mathrm{Fe})$ by solvothermal method and applied them as a catalyst for photochemical degradation of organic pigments Rhodamine $\mathrm{B}(\mathrm{RhB})$ in water under visible light. The synthesized materials were studied for structural characterization by methods of X-ray diffraction (XRD), ultravioletvisible spectroscopy (UV-VIS), scanning electron microscope (SEM), Fourier-transform infrared spectroscopy (FT-IR), $\mathrm{N}_{2}$ adsorptiondesorption analysis using BET method. The degradation efficiency was evaluated through the ability of photocatalyst using visible light to degrade $\mathrm{RhB}$ dyes on synthetic materials under the assumed light of $40 \mathrm{~W}$ LED. The main influencing factors, such as: $\mathrm{pH}$, initial colorant concentration, catalyst mass, and the activity of the main oxidants affecting the degradation efficiency of organic pigments, were also studied. On the other hand, the catalytic stability, as well as the ability to regenerate and reuse the catalyst, were also evaluated, thereby suggesting the mechanism of action for the amount of $\mathrm{Mn} / \mathrm{Fe}-\mathrm{MOF}$ in the photocatalysis process degrade $\mathrm{RhB}$.

\section{Materials and Methods}

\subsection{Chemicals}

The main chemicals used in this study include: Iron(III) nitrate nonahydrate $\mathrm{Fe}\left(\mathrm{NO}_{3}\right)_{3} .9 \mathrm{H}_{2} \mathrm{O}$ (China 98.5\%), Manganese Chloride $\mathrm{MnCl}_{2} .6 \mathrm{H}_{2} \mathrm{O}$ (China 99\%), Acetonitrile $\mathrm{CH}_{3} \mathrm{CN}$ (Merck, Germany 99.5\%) Terephthalic acid $\mathrm{H}_{2} \mathrm{BDC}$ (Sigma Aldrich 98\%), Meth- 
anol $\mathrm{CH}_{3} \mathrm{OH}$ (China), N,N-dimethylfomamide DMF (China 99.5\%).

\subsection{Synthesis of Mn/Fe-MOF}

The organic hybrid $0.1 \mathrm{Mn} / \mathrm{Fe}-\mathrm{MOF}$ material was prepared based on the synthesis process of Fe-MOF metal-mechanical framework material described in the literature [28,29]. The mixture consists of $0.6 \mathrm{~g}$ of $\mathrm{Fe}\left(\mathrm{NO}_{3}\right)_{3} .9 \mathrm{H}_{2} \mathrm{O}$ $(0.1455 \mathrm{mmol}), 0.03 \mathrm{~g}$ of $\mathrm{MnCl}_{2} .6 \mathrm{H}_{2} \mathrm{O}(0.0145$ $\mathrm{mmol}$ ) and $0.3 \mathrm{~g}$ of 1,4 benzene dicarboxylic acid $(0.18 \mathrm{mmol})$ and $40 \mathrm{~mL}$ of $\mathrm{N}^{\prime} \mathrm{N}$ dimethylformamide - DMF. Next, $40 \mathrm{~mL}$ $\mathrm{CH}_{3} \mathrm{CN}$ was added to the stirring solution at room temperature for $30 \mathrm{~min}$. The mixture was then placed in a $100 \mathrm{~mL}$ Teflon flask (with stainless steel cover) and placed in an oven at $150{ }^{\circ} \mathrm{C}$ for $12 \mathrm{~h}$. After cooling to room temperature, the suspension was centrifuged to obtain the solid product (orange powder) in the Teflon tube. The material was further washed 3 times with DMF and 3 times with methanol. After cleaning, the material was dried overnight at $150^{\circ} \mathrm{C}$.

\subsection{Characterization}

The crystalline phase composition of the material was determined by X-ray diffraction (XRD) and measured on a D8 Advanced - Hitachi (Germany). FT-IR infrared spectroscopy analysis method used to determine functional groups, identify organic compounds, and study the structure, was measured on Nicolet 6700 Thermo Fisher Scientific (USA). The structure and surface morphology of the samples in this study will be investigated through SEM image

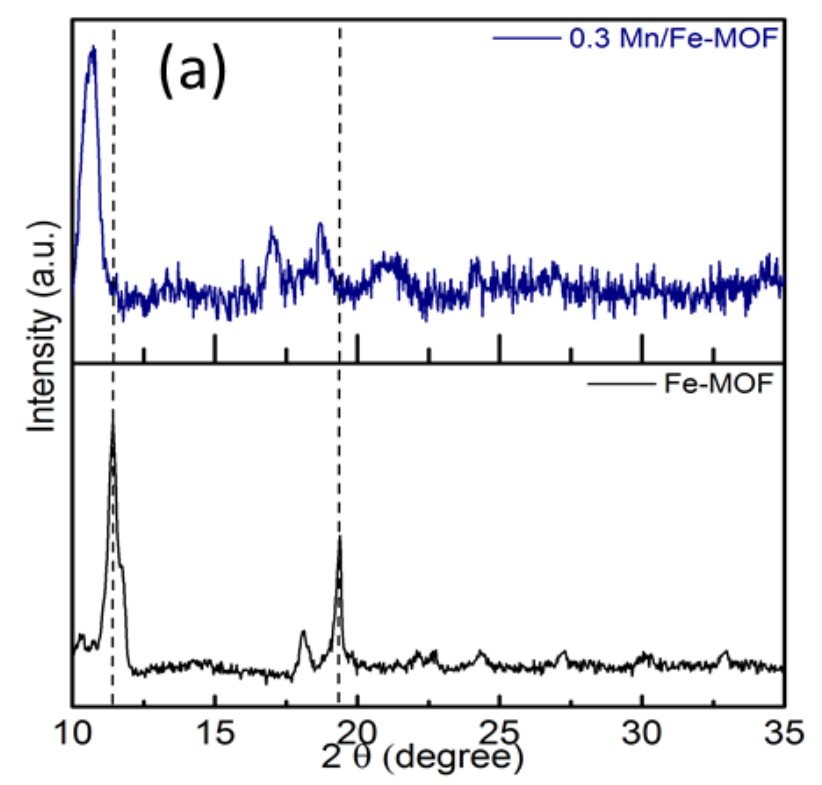

S4800 - JEOL (Japan). In this study, a specific surface area determined by nitrogen adsorption-desorption isotherm was performed on Micromeritics 2020 - Micromeritics, USA. Ultraviolet and visible spectroscopy methods were used to determine the content of dyes in aqueous solutions and were performed on a UV-Vis spectrometer recorded on a Cary60 UV-Visible Spectrophotometer.

\subsection{Photocatalytic Test}

The photocatalytic activity of $0.1 \mathrm{Mn} / \mathrm{Fe}$ MOF was evaluated based on the degradation efficiency of $\mathrm{RhB}$ under visible light irradiation. Performance conditions at room temperature, the light source used is a 40W LED. RhB concentrations were analyzed using a UVvisible spectrophotometer (Model Evolution 60S, Thermo Fisher Scientific, Waltham, MA, USA) at a maximum absorption wavelength $\lambda$ $=554 \mathrm{~nm}$. The reaction system was carried out including $5 \mathrm{mg}$ of catalyst sample, $100 \mathrm{~mL}$ of RhB solution $\left(3 \times 10^{-5} \mathrm{M}\right)$ and $1 \mathrm{~mL}$ of $\mathrm{H}_{2} \mathrm{O}_{2}$ solution $(1 \mathrm{mM})$ in a $250 \mathrm{~mL}$ beaker. Besides, the parameters affecting the degradation of $\mathrm{RhB}$ colorants including initial dye concentration, initial solution $\mathrm{pH}$ and catalyst content were also studied.

\section{Results and Discussion}

\subsection{Synthesis and Characterization}

The topology and crystal structure of the Fe-MOF and $0.1 \mathrm{Mn} / \mathrm{Fe}-\mathrm{MOF}$ samples characterized by the diffraction peak is clearly and sharply resolved in Figure 1(a). The results

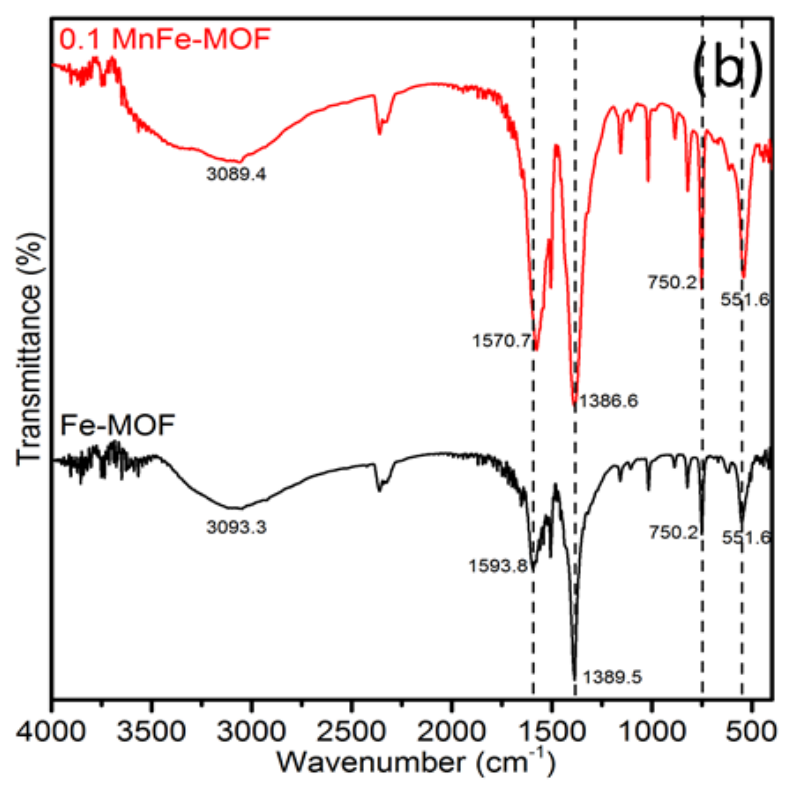

Figure 1. XRD (a) and FT-IR (b) diagram of Fe-MOF and 0.1Mn/Fe-MOF. 
show that the growth of the well-crystallized MOF structure, the Fe-MOF sample showed characteristic diffraction peaks at $2 \theta$ of $11.42^{\circ}$; $18.13^{\circ} ; 19.38^{\circ}$ corresponds to the study of Sun et al. [28]. The Mn-modified Fe-MOF samples after X-ray diffraction (XRD) analysis both displayed similar characteristic peaks, indicating that they have nearly identical crystal structures, although there are some slight differences in their properties peak intensity. However, magnifying at about $10^{\circ}-20^{\circ}$, the diffraction peak of about $11.42^{\circ}$ is clearly shifted to a higher angle at $0.1 \mathrm{Mn} / \mathrm{Fe}-\mathrm{MOF}$ molar ratio. The Mn/Fe-MOF diffraction peaks are less intense and more extensive than that of the $\mathrm{Fe}$ MOF sample, indicating that the $0.1 \mathrm{Mn} / \mathrm{Fe}$ MOF sample has a relatively lower crystallinity [24].

FT-IR spectroscopy was used to determine the molecular structure and functional groups of the Fe-MOFs and $0.1 \mathrm{Mn} / \mathrm{Fe}-\mathrm{MOF}$ (Figure 1(b)). The characteristic peaks at $3093.3 \mathrm{~cm}^{-1}$ characterize the existence of $\mathrm{O}-\mathrm{H}$ bonding groups of water molecules adsorbed onto the
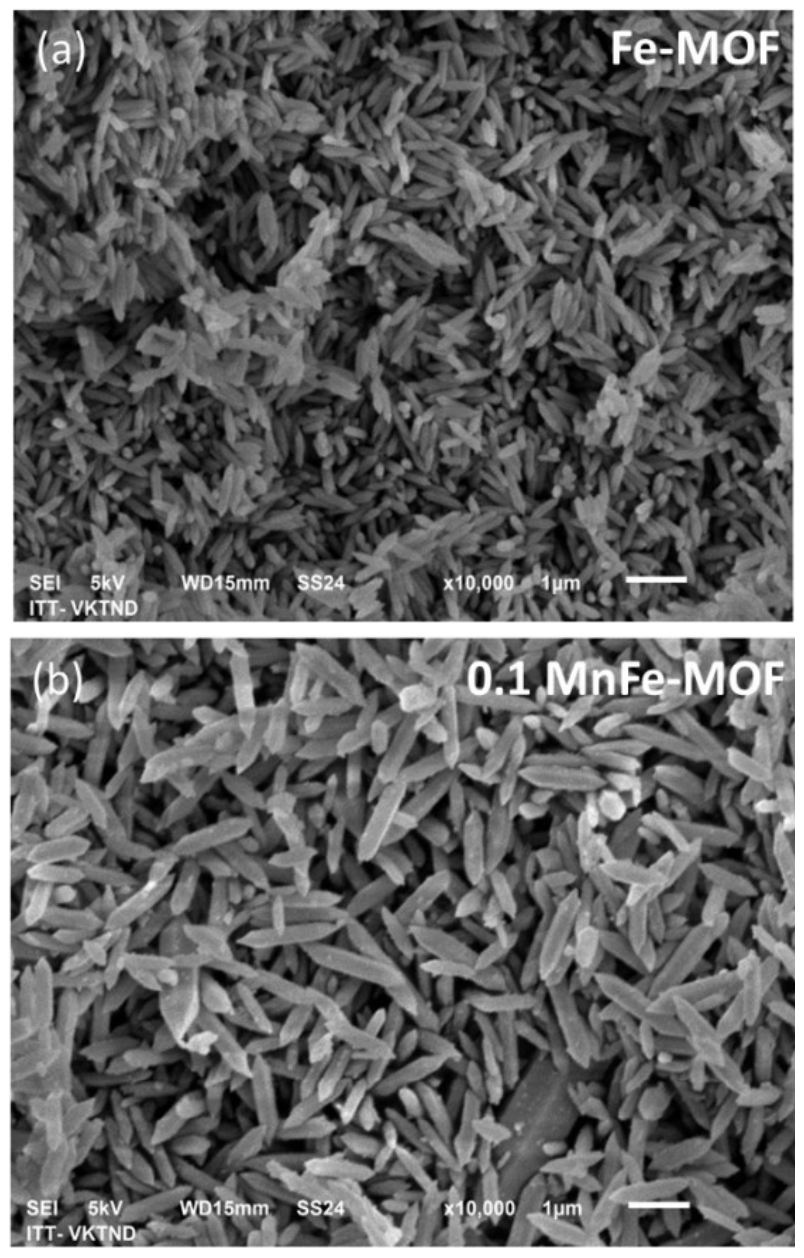

Figure 2. SEM images of Fe-MOF and $0.1 \mathrm{Mn} / \mathrm{Fe}-\mathrm{MOF}$. surface of the material. A wavenumber 1593.8, $1389.5 \mathrm{~cm}^{-1}$ is typical for symmetric and asymmetric valence vibration of the carboxylic group of BDC, the absorption band at wave number $750.2 \mathrm{~cm}^{-1}$ is characteristic for $\mathrm{C}-\mathrm{H}$ bond vibration of the benzene ring. In addition, the $551.6 \mathrm{~cm}^{-1}$ peak characterizes the oscillation of the $\mathrm{Fe}-\mathrm{O}$ bond in the spectrum of $\mathrm{Fe}-\mathrm{MOF}$. However, the corresponding peaks in the $0.1 \mathrm{Mn} / \mathrm{Fe}-\mathrm{MOF}$ FT-IR spectrum were determined to be $3089.4,1570.7,1386.6,750.2$, and $551.6 \mathrm{~cm}^{-1}$, respectively, the sample peak intensity $0.1 \mathrm{Mn} / \mathrm{Fe}-\mathrm{MOF}$ at large characteristic wavenumbers than Fe-MOF, although there was no change between the peak-to-peak binding oscillations.

Figure 2 Surface morphology of Fe-MOFs observed by SEM shows that the typical grain size of Fe-MOFs is mainly the formation of well-defined hexagonal bars with pointed tips. However, the shape and size of the Fe-MOF
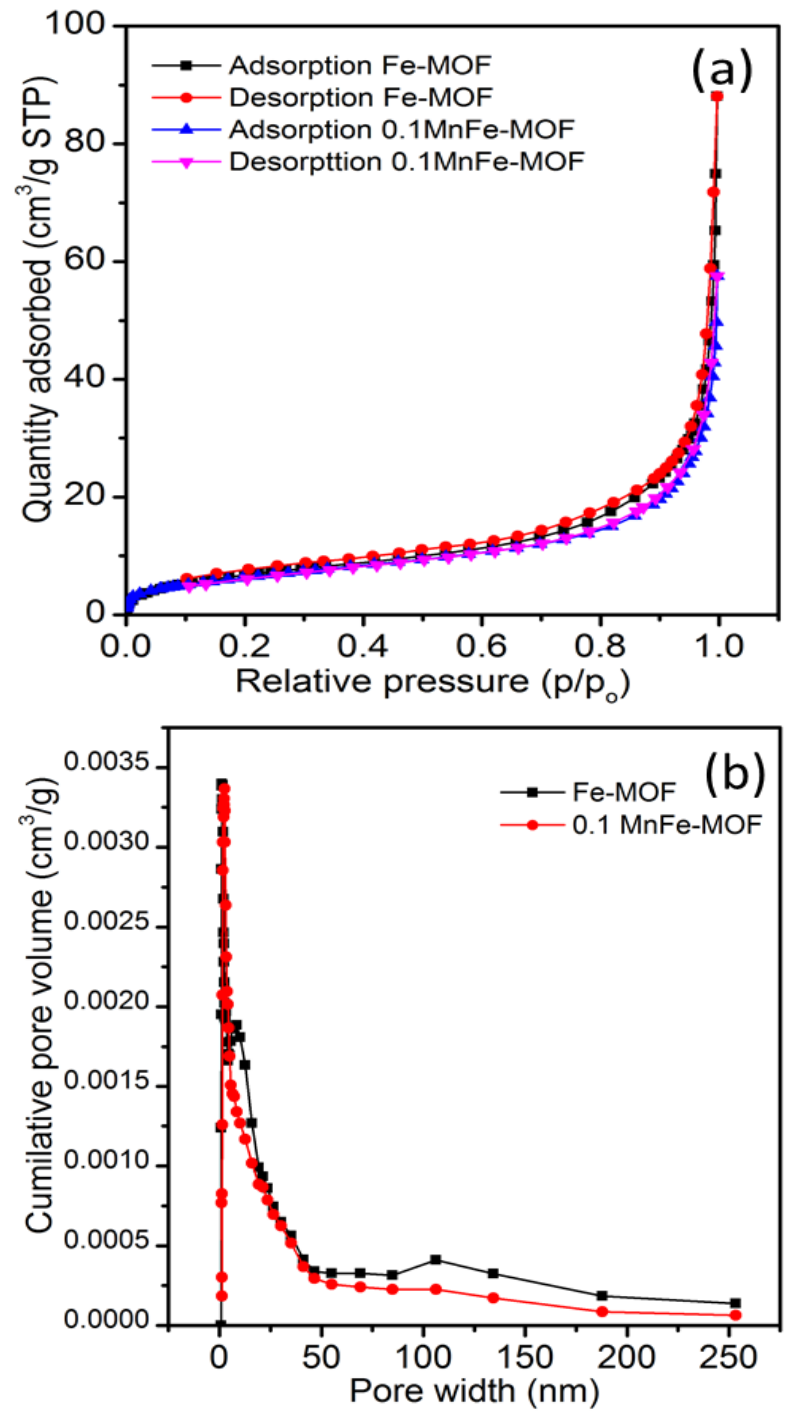

Figure 3. Nitrogen adsorption-desorption isotherms of Fe-MOF and 0.1 Mn/Fe-MOF. 
modified materials changed markedly in the presence of $\mathrm{Mn}$ metal at a $0.1 \mathrm{MnFe}$ molar ratio. When increasing the $0.1 \mathrm{MnFe} \mathrm{MOF}$ ratios, the particles are homogeneous and there is an increase in size and shape. Research results show that $\mathrm{Fe}-\mathrm{MOF}$ is consistent with previous publications [22].

The average capillary structure of Fe-MOF and $0.1 \mathrm{Mn} / \mathrm{Fe}-\mathrm{MOF}$ materials was confirmed through the Nitrogen adsorption-desorption isotherm (BET) study (Figure 3). We found that the Nitrogen adsorption-desorption curves of the materials all belong to type IV adsorption isotherms (according to IUPAC classification) which is typical for the presence of medium capillary type. The surface area (BET) and pore size of the Fe-MOF material are $25.68 \mathrm{~m}^{2} / \mathrm{g}$ and the pore size is $13.56 \mathrm{~nm}$, respectively. Meanwhile, the $0.1 \mathrm{Mn} / \mathrm{Fe}-\mathrm{MOF}$ sample has a surface area (BET) of $23.57 \mathrm{~m}^{2} / \mathrm{g}$ and pore size of 11.37 $\mathrm{nm}$. Compared with the published results on specific surface area and pore diameter of $\mathrm{Fe}$ MOF materials published by Zhao et al. [36] are $42.11 \mathrm{~m}^{2} / \mathrm{g}$ and $11.40 \mathrm{~nm}$. This difference is
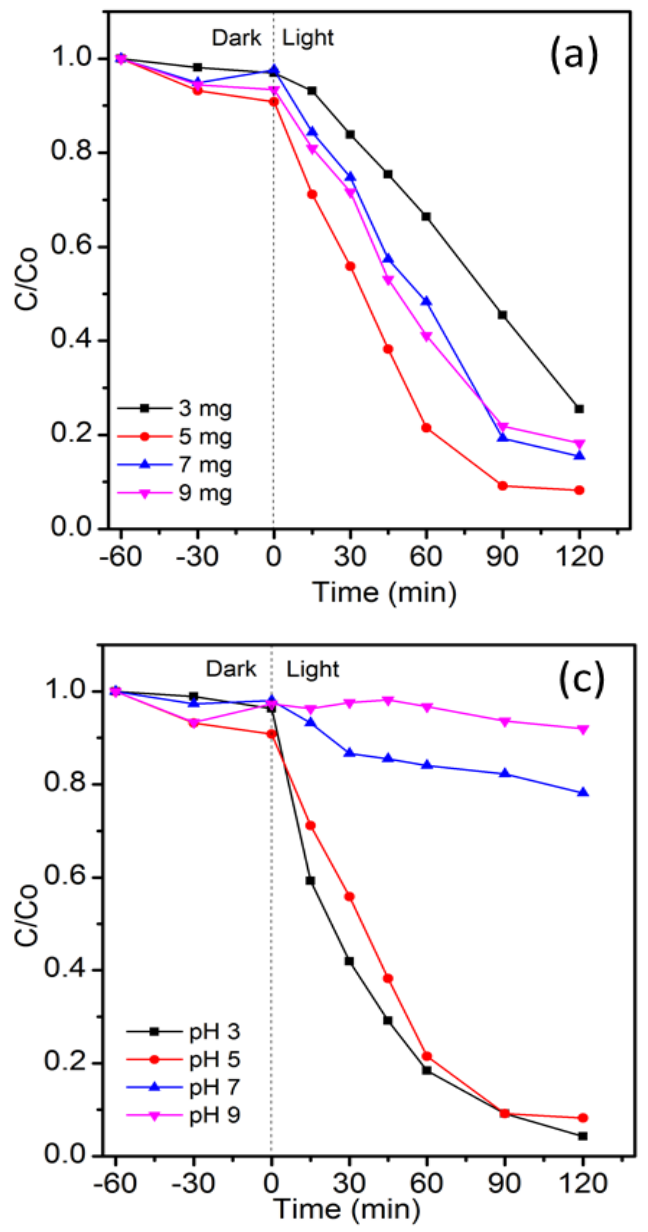

probably explained by different synthesis conditions, so the crystal structure of the obtained materials is different, and therefore the specific surface area is different [31].

\subsection{Photocatalytic Activities}

The influence of key reaction parameters on $\mathrm{RhB}$ degradation efficiency in a typical 0.1 $\mathrm{Mn} / \mathrm{Fe}-\mathrm{MOF} / \mathrm{H}_{2} \mathrm{O}_{2}$ reaction system such as $\mathrm{RhB}$ colorant concentration, $\mathrm{pH}$, and amount of catalyst were studied. In the Fenton-like system, $\mathrm{H}_{2} \mathrm{O}_{2}$ is considered as a direct participant in the formation of hydroxyl radicals.

Experimental results in Figure 4(a) show that the change of catalyst content from $3 \mathrm{mg}$ to $5 \mathrm{mg}$ leads to an increase in $\mathrm{RhB}$ decomposition efficiency, which increases, respectively from $74.35 \%$ to $91.78 \%$ after 120 minutes of irradiation. However, when the amount of photocatalyst is increased further $(7 \mathrm{mg}$ and $9 \mathrm{mg}$ ), the degradation efficiency tends to decrease because the excess catalyst can scatter the photons in the photoreaction system. Usually, an increase in the number of photocatalysts leads
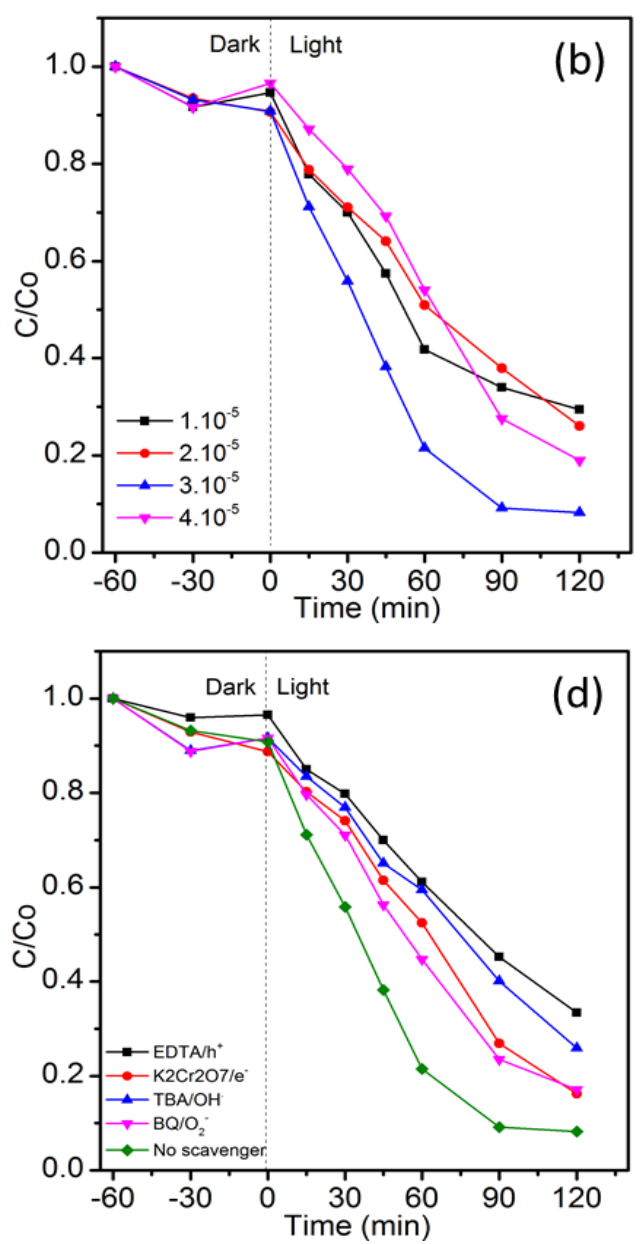

Figure 4. The influence of catalyst mass $0.1 \mathrm{Mn} / \mathrm{Fe}-\mathrm{MOF}$ (a), initial dye concentration (b), initial solution $\mathrm{pH}(\mathrm{c})$, and different scavengers (d) on the degradation of RhB. 
to an increase in photocatalytic activity, more or less dependent on the configuration and reaction conditions [32]. However, there is a saturation effect, and beyond this point, a decrease in degradation efficiency is observed as the amount of photocatalyst is increased, usually due to the light blocking created by the increase in water turbidity due to a large number of suspended particles of the photocatalyst [33].

The influence of the initial pigment concentration was investigated in the range of $1 \times 10^{-5}$ - $4 \times 10^{-5} \mathrm{M}$. Results of Figure 4(b) shows the impact of $\mathrm{RhB}$ concentration on the photocatalytic degradation of $0.1 \mathrm{Mn} / \mathrm{Fe}-\mathrm{MOF}$, with $\mathrm{RhB}$ concentration $3 \times 10^{-5} \mathrm{M}$ after 120 minutes, the decomposition efficiency was $91.78 \%$. However, when continuing to increase the $\mathrm{RhB}$ concentration to $4 \times 10^{-5} \mathrm{M}$, the efficiency decreased significantly to only $81.03 \%$. The degradation efficiency at higher concentrations is due to the absence of photoactive sites due to increased adsorption of dye molecules and decreased speed of light entering the suspension.

The effect of the initial $\mathrm{pH}$ value of $\mathrm{RhB}$ degradation in an aqueous medium is shown by the initial $\mathrm{pH}$ value of the solution from 3 to 9 . Figure 4(c) shows that the rate of RhB degradation in the acidic medium $(\mathrm{pH} 3-5)$ is much higher than in the basic medium ( $\mathrm{pH} 7-9)$. Indeed, the degradation efficiency was reduced from $94.75 \%$ to $8 \%$ when increasing the initial $\mathrm{pH}$ from 3 to 9 . Besides, the RhB color used in this experiment is a cationic color, so the material will absorb the color increasing from $\mathrm{pH} 5$ to 9. As absorption increases, the color molecules will shield the catalyst surface, resulting in prevents light from irradiating onto the catalyst surface, so the photocatalytic activity is reduced and the color removal ability is reduced. On the other hand, because $\mathrm{H}_{2} \mathrm{O}_{2}$ is unstable in alkaline media, it decomposes into $\mathrm{H}_{2} \mathrm{O}$ and $\mathrm{O}_{2}$. Since the surface of $\mathrm{RhB}$ is positively charged, photovoltaic electrons can transfer to the sur- face of the particle and enter the molecular structure of $\mathrm{RhB}$, leading to the decomposition of RhB. In addition, in photocatalysis, holes and the number of photogenerated electrons approaching the surface of the particles will determine the efficiency of dye degradation [34]. In strongly acidic $\mathrm{pH}, \mathrm{RhB}$ exists in higher concentrations at the bubble interface and is more susceptible to attack by the $\mathrm{OH}$ radical. Factors such as acid-base properties, ionization state of surface particles, and reactive dyes are among the factors affecting $\mathrm{pH}$ that directly affect the photodegradation efficiency of dyes.

Various electron capturers used for the determination of major oxidants include 1,4-benzoquinone (BQ) used as superoxide anionic radical scavenger $\left(\mathrm{O}_{2} \cdot{ }^{-}\right)$, potassium dichromate $\left(\mathrm{K}_{2} \mathrm{Cr}_{2} \mathrm{O}_{7}\right)$, and ethylenediaminetetraacetic acid (EDTA) as a determiner of electrons $\left(e^{-}\right)$and holes $\left(h^{+}\right)$, and tert-butanol (TBA) was selected as the hydroxyl radical scavenger for all $\cdot \mathrm{OH}$ formed during the reaction $[35,36]$. The experimental results are shown in Figure 4(d), the decomposition efficiency changes markedly in the presence of electron-capturing substances in the reaction system. It is noteworthy that the dye degradation was significantly reduced in the presence of EDTA and TBA found that $h^{+}$and $\mathrm{O}_{2}{ }^{-}$ played a major role as free radical oxidants. Whilst $\mathrm{BQ}$ and $\mathrm{K}_{2} \mathrm{Cr}_{2} \mathrm{O}_{7}$ were added to the mixed reaction, the dye concentration and color intensity were slightly changed, indicating that in the photochemical decomposition there is the activity of superoxide anion and $e^{-}$radicals.

Based on the proposed mechanism in Figure 5 , with the enlarged surface area and pore volume of Mn-doped bimetallic MOFs, adsorption sites inside or on the surface of the framework are available to adsorb more dye molecules in solution. The $\mathrm{Mn} / \mathrm{Fe}-\mathrm{MOF}$ material is excited by ultraviolet light and an electron $\left(e^{-}\right)$- hole $\left(\mathrm{h}^{+}\right)$pair on its surface is formed. After the

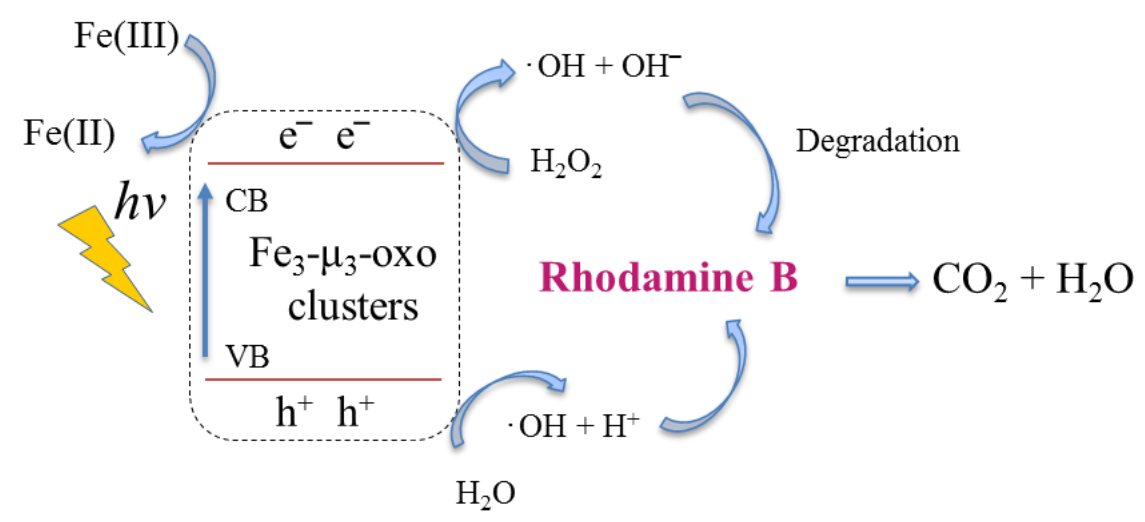

Figure 5. Proposed mechanism of $0.1 \mathrm{Mn} / \mathrm{Fe}-\mathrm{MOF}$. 
MOF absorbs energy, the substance is excited from the valence band (VB) and enters the conduction band (CB), leaving $\mathrm{h}^{+}$in the VB. The $\mathrm{e}^{-}$ and $\mathrm{h}^{+}$migrate to the MOF surface, then the electrons reduce the oxygen molecule to oxygen radicals $\left(\mathrm{O}_{2} \cdot-\right)$, and finally, they convert to hydroxyl radicals $(\mathrm{OH} \bullet)$. In addition, the $\mathrm{h}^{+}$oxidized the water molecule to $\mathrm{OH} \cdot$ the hydroxyl radicals effectively degrade the dye.

An experiment was performed under optimal conditions by using the "leaching" experiment. The results in Figure 6(a) show that the reaction occurs very quickly in the presence of the $0.1 \mathrm{Mn} / \mathrm{Fe}-\mathrm{MOF}$ catalysts. In contrast, when removing the catalyst after $30 \mathrm{~min}$ of irradiation, most $\mathrm{RhB}$ concentrations were slow degradation and there was no conversion after 120 min. Photochemical degradation took place only in the presence of a solid $0.1 \mathrm{Mn} / \mathrm{Fe}-\mathrm{MOF}$ catalyst and demonstrated that the catalytic sample was insoluble in solution, although the presence of dissolved co-activators in solution was negligible significantly.

In practical applications of catalysts, recyclability is an important factor. The possibility of $\mathrm{Mn} / \mathrm{Fe}-\mathrm{MOF}$ reuse was also studied through the catalytic loop in the $\mathrm{RhB}$ degradation reaction of $0.1 \mathrm{Mn} / \mathrm{Fe}-\mathrm{MOF} / \mathrm{lamp}$ irradiation $/ \mathrm{H}_{2} \mathrm{O}_{2}$. As shown in Figure 6(b), although the RhB removal efficiency decreased slightly as the catalyst cycle was increased (5 times), acceptable catalytic activity was maintained, the $\mathrm{RhB}$ degradation efficiency was still achieved over $83 \%$. The $0.1 \mathrm{Mn} / \mathrm{Fe}-\mathrm{MOF}$ modified material exhibits high stability and recyclability for the degradation of $\mathrm{RhB}$ organic pigments, offering great potential for the practical treatment of pollutants.

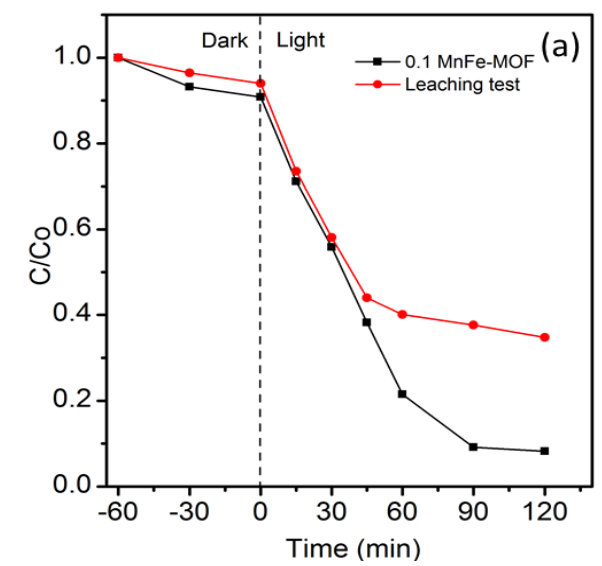

\section{Conclusions}

The bimetallic organic framework $\mathrm{Mn} / \mathrm{Fe}$ MOF has been successfully synthesized with regular crystal distribution, rather high crystalline intensity consistent with XRD results. The optimal conditions in this study for the successful synthesis of the material were at the hydrothermal temperature of $150{ }^{\circ} \mathrm{C}$, the hydrothermal time of 12 hours. The synthesized $0.1 \mathrm{Mn} / \mathrm{Fe}-\mathrm{MOF}$ sample has a hexagonal structure with a relatively uniform, smooth surface and sharp edges, but inside contains medium capillaries with a hole-like structure with a diameter of $11.3719 \mathrm{~nm}$, The specific surface area of the composite sample is $23.5704 \mathrm{~m}^{2} / \mathrm{g}$. The $0.1 \mathrm{Mn} / \mathrm{Fe}-\mathrm{MOF}$ material has catalytic activity for $\mathrm{RhB}$ decomposition of about $92 \%$ and has high reusability, the activity after 5 reuses is up to $84 \%$ for the $\mathrm{RhB}$ decomposition reaction. From there, opening research directions in application in the field of adsorption or catalysis in the treatment of toxic colorants from mixed metal MOFs.

\section{Acknowledgment}

This study was supported by grants from Nguyen Tat Thanh University, Ho Chi Minh City, Viet Nam.

\section{References}

[1] Abednatanzi, S., Gohari Derakhshandeh, P., Depauw, H., Coudert, F.X., Vrielinck, H., Van Der Voort, P., Leus, K. (2019). Mixed-metal metal-organic frameworks. Chemical Society Reviews, 48(9), 2535-2565. DOI: $10.1039 / \mathrm{c} 8 \mathrm{cs} 00337 \mathrm{~h}$

[2] Alwash, A.H., Abdullah, A.Z., Ismail, N. (2012). Zeolite Y encapsulated with Fe-TiO2 for ultrasound-assisted degradation of amaranth dye in water. Journal of Hazardous Materials, 233-234, 184-193. DOI: 10.1016/j.jhazmat.2012.07.021

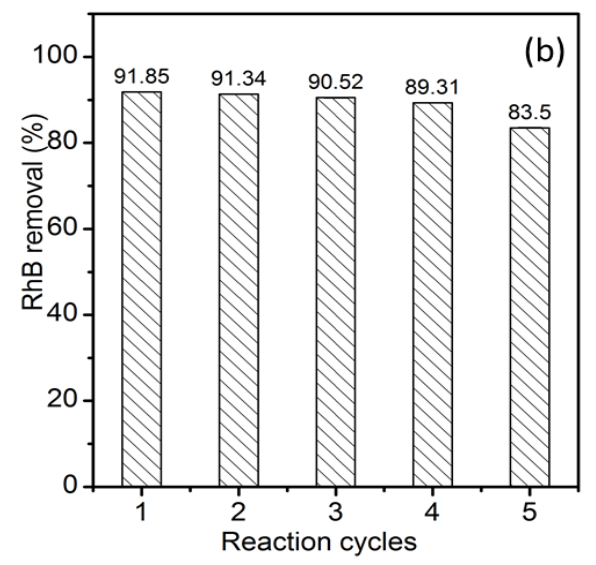

Figure 6. Leaching (a) and recycling (b) test of the $0.1 \mathrm{Mn} / \mathrm{Fe}-\mathrm{MOF}$ catalyst material. 
[3] Calleja, G., Sanz, R., Orcajo, G., Briones, D., Leo, P., Martínez, F. (2014). Copper-based MOF-74 material as effective acid catalyst in Friedel-Crafts acylation of anisole. Catalysis Today, 227, $130-137$. D O I : 10.1016/j.cattod.2013.11.062

[4] Chen, L., Wang, H.F., Li, C., Xu, Q. (2020). Bimetallic metal-organic frameworks and their derivatives. Chemical Science, 11(21), 5369-5403. DOI: 10.1039/d0sc01432j

[5] Choi, S., Cha, W., Ji, H., Kim, D., Lee, H. J., Oh, M. (2016). Synthesis of hybrid metalorganic frameworks of $\left\{\mathrm{Fe}: \mathrm{XMyM}^{\prime} 1-\mathrm{x}-\mathrm{Y}\right\}$ MIL-88B and the use of anions to control their structural features. Nanoscale, 8(37), 16743-16751. DOI: 10.1039/c6nr05463c

[6] Gao, Y., Li, S., Li, Y., Yao, L., Zhang, H. (2017). Accelerated photocatalytic degradation of organic pollutant over metal-organic framework MIL-53(Fe) under visible LED light mediated by persulfate. Applied Catalysis B: Environmental, 202, 165-174. DOI: 10.1016/j.apcatb.2016.09.005

[7] Gholizadeh Khasevani, S., Gholami, M.R. (2019). Evaluation of the reaction mechanism for photocatalytic degradation of organic pollutants with MIL-88A/BiOI structure under visible light irradiation. Research on Chemical Intermediates, 45(3), 1341-1356. DOI: 10.1007/s11164-018-3681-9

[8] Gu, Y., Xie, D., Wang, Y., Qin, W., Zhang, H., Wang, G., Zhang, Y., Zhao, H. (2019). Facile fabrication of composition-tunable $\mathrm{Fe} / \mathrm{Mg}$ bimetal-organic frameworks for exceptional arsenate removal. Chemical Engineering Journal, $357, \quad 579-588$. D O I : 10.1016/j.cej.2018.09.174

[9] Haque, E., Lee, J.E., Jang, I.T., Hwang, Y.K., Chang, J.S., Jegal, J., Jhung, S.H. (2010). Adsorptive removal of methyl orange from aqueous solution with metal-organic frameworks, porous chromium-benzenedicarboxylates. Journal of Hazardous Materials, 181(1-3), 535-542. DOI: 10.1016/j.jhazmat.2010.05.047

[10] Hong, D.Y., Hwang, Y.K., Serre, C., Férey, G., Chang, J.S. (2009). Porous chromium terephthalate MIL-101 with coordinatively unsaturated sites: Surface functionalization, encapsulation, sorption and catalysis. Advanced Functional Materials, 19(10), 1537-1552. DOI: $10.1002 / \mathrm{adfm} .200801130$

[11] Khan, N.A., Jhung, S.H. (2013). Effect of central metal ions of analogous metal-organic frameworks on the adsorptive removal of benzothiophene from a model fuel. Journal of Hazardous Materials, 260, 1050-1056. DOI: 10.1016/j.jhazmat.2013.06.076
[12] Khan, N.A., Jhung, S.H. (2015). Synthesis of metal-organic frameworks (MOFs) with microwave or ultrasound: Rapid reaction, phaseselectivity, and size reduction. Coordination Chemistry Reviews, 285, 11-23. DOI: 10.1016/j.ccr.2014.10.008

[13] Koh, K., Wong-Foy, A.G., Matzger, A.J. (2009). A porous coordination copolymer with over $5000 \mathrm{~m} \mathrm{2/g} \mathrm{BET} \mathrm{surface} \mathrm{area.} \mathrm{Journal} \mathrm{of}$ the American Chemical Society, 131(12), 4184-4185. DOI: 10.1021/ja809985t

[14] Kuppler, R.J., Timmons, D.J., Fang, Q.R., Li, J.R., Makal, T.A., Young, M.D., Yuan, D., Zhao, D., Zhuang, W., Zhou, H.C. (2009). Potential applications of metal-organic frameworks. Coordination Chemistry Reviews, 253(23-24), 3042-3066. DO I : 10.1016/j.ccr.2009.05.019

[15] Lebedev, O.I., Millange, F., Serre, C., Van Tendeloo, G., Férey, G. (2005). First direct imaging of giant pores of the metal-organic framework MIL-101. Chemistry of Materials, 17(26), 6525-6527. DOI: 10.1021/cm051870o

[16] Merouani, S., Hamdaoui, O., Saoudi, F., Chiha, M. (2010). Sonochemical degradation of Rhodamine B in aqueous phase: Effects of additives. Chemical Engineering Journal, $158(3), \quad 550-557$. D O I : 10.1016/j.cej.2010.01.048

[17] Nguyen, H.T.T., Dinh, V.P., Phan, Q.A.N., Tran, V.A., Doan, V.D., Lee, T., Nguyen, T.D. (2020). Bimetallic Al/Fe Metal-Organic Framework for highly efficient photo-Fenton degradation of rhodamine B under visible light irradiation. Materials Letters, 279, 128482. DOI: 10.1016/j.matlet.2020.128482

[18] Nguyen, L.T.L., Nguyen, C.V., Dang, G.H., Le, K.K.A., Phan, N.T.S. (2011). Towards applications of metal-organic frameworks in catalysis: Friedel-Crafts acylation reaction over IRMOF-8 as an efficient heterogeneous catalyst. Journal of Molecular Catalysis A: Chemical, $349(1-2), \quad 28-35$. DOI : 10.1016/j.molcata.2011.08.011

[19] Nguyen, V.H., Nguyen, T.D., Bach, L.G., Hoang, T., Bui, Q.T.P., Tran, L.D., Nguyen, C.V., Vo, D.-V.N., Do, S.T. (2018). Effective Photocatalytic Activity of Mixed Ni/Fe-Base Metal-Organic Framework under a Compact Fluorescent Daylight Lamp. Catalysts, 8(11), 487. DOI: $10.3390 /$ catal 8110487

[20] Pham, M.H., Vuong, G.T., Vu, A.T., Do, T.O. (2011). Novel route to size-controlled Fe-MIL88B-NH 2 metal-organic framework nanocrystals. Langmuir, 27(24), 15261-15267. DOI: $10.1021 / \mathrm{la} 203570 \mathrm{~h}$ 
[21] Qasem, N.A.A., Ben-Mansour, R., Habib, M.A. (2018). An efficient $\mathrm{CO}_{2}$ adsorptive storage using MOF-5 and MOF-177. Applied Energy, $210, \quad 317-326$. D O I : 10.1016/j.apenergy.2017.11.011

[22] Tang, J., Wang, J. (2020). Iron-copper bimetallic metal-organic frameworks for efficient Fenton-like degradation of sulfamethoxazole under mild conditions. Chemosphere, 241, 12 $\begin{array}{lllllllll}5 & 0 & 0 & 2 & \text {. } & \text { D } & \text { O } & \text { I }\end{array}$ 10.1016/j.chemosphere.2019.125002

[23] Qu, L.L., Wang, J., Xu, T.Y., Chen, Q.Y., Chen, J.H., Shi, C.J. (2018). Iron(III)-based metal-organic frameworks as oxygen-evolving photocatalysts for water oxidation. Sustainable Energy and Fuels, 2(9), 2109-2114. DOI: $10.1039 / \mathrm{c} 8 \mathrm{se} 00311 \mathrm{~d}$

[24] Sun, Q., Liu, M., Li, K., Han, Y., Zuo, Y., Chai, F., Song, C., Zhang, G., Guo, X. (2017). Synthesis of $\mathrm{Fe} / \mathrm{M}(\mathrm{M}=\mathrm{Mn}, \mathrm{Co}, \mathrm{Ni})$ bimetallic metal organic frameworks and their catalytic activity for phenol degradation under mild conditions. Inorganic Chemistry Frontiers, 4(1), 144-153. DOI: 10.1039/C6QI00441E

[25] Rahmani, E., Rahmani, M. (2017). Alkylation of benzene over Fe-based metal organic frameworks (MOFs) at low temperature condition. Microporous and Mesoporous Materials, $249, \quad 118-127$. D O I : 10.1016/j.micromeso.2017.04.058

[26] Rowsell, J.L.C., Yaghi, O.M. (2004). Metalorganic frameworks: A new class of porous materials. Microporous and Mesoporous Materials, $73(1-2), \quad 3-14$. D O : 10.1016/j.micromeso.2004.03.034

[27] Rowsell, J.L.C., Yaghi, O.M. (2005). Strategies for hydrogen storage in metal-organic frameworks. Angewandte Chemie - International Edition, 44(30), 4670-4679. DOI: 10.1002/anie.200462786

[28] Saleh, R., Djaja, N.F. (2014). UV light photocatalytic degradation of organic dyes with $\mathrm{Fe}$ doped $\mathrm{ZnO}$ nanoparticles. Superlattices and Microstructures, 74, 217-233. DOI: 10.1016/j.spmi.2014.06.013

[29] Su, S., Guo, W., Leng, Y., Yi, C., Ma, Z. (2013). Heterogeneous activation of Oxone by CoxFe3-xO4 nanocatalysts for degradation of rhodamine B. Journal of Hazardous Materials, 244-245, 736-742. DOI: 10.1016/j.jhazmat.2012.11.005
[30] Geng, N., Chen, W., Xu, H., Ding, M., Lin, T., $\mathrm{Wu}, \mathrm{Q}$., Zhang, L. (2021). Insights into the novel application of Fe-MOFs in ultrasoundassisted heterogeneous Fenton system: Efficiency, kinetics and mechanism. Ultrasonics Sonochemistry, 72, 105411. DOI: 10.1016/j.ultsonch.2020.105411

[31] Wang, D., Albero, J., García, H., Li, Z. (2017). Visible-light-induced tandem reaction of oaminothiophenols and alcohols to benzothiazoles over Fe-based MOFs: Influence of the structure elucidated by transient absorption spectroscopy. Journal of Catalysis, 349, 156162. DOI: 10.1016/j.jcat.2017.01.014

[32] Wang, D., Li, Z. (2017). Iron-based metalorganic frameworks (MOFs) for visible-lightinduced photocatalysis. Research on Chemical Intermediates, 43(9), 5169-5186. DOI: 10.1007/s11164-017-3042-0

[33] Wang, J., Zhang, M., Li, S., Liu, R., Li, Z. (2020). Metal-organic frameworks-derived hollow-structured iron-cobalt bimetallic phosphide electrocatalysts for efficient oxygen evolution reaction. Journal of Alloys and Compounds, $821, \quad 153463$. DO I : 10.1016/j.jallcom.2019.153463

[34] Wang, S., Yang, Q., Zhong, C. (2008). Adsorption and separation of binary mixtures in a metal-organic framework Cu-BTC: A computational study. Separation and Purification Technology, 60(1), 30-35. DOI: 10.1016/j.seppur.2007.07.050

[35] Wu, Q., Siddique, M.S., Yu, W. (2021). Ironnickel bimetallic metal-organic frameworks as bifunctional Fenton-like catalysts for enhanced adsorption and degradation of organic contaminants under visible light: Kinetics and mechanistic studies. Journal of Hazardous Materials, 401, 123261. DOI: 10.1016/j.jhazmat.2020.123261

[36] Zhao, Y., Wang, H.L., Qi, H.P., Zhao, D.Y., Wang, X.K. (2020). Facile synthesis of low crystalline BiOCl-based thermally-responsive photocatalyst with enhanced catalytic performance for photodegrading rhodamine B solution. Journal of Alloys and Compounds, 819, 153042. DOI: 10.1016/j.jallcom.2019.153042 